\title{
Erratum
}

\section{Cyclodextrins: A Weapon in the Fight Against Antimicrobial Resistance}

\author{
[J. Molecular and Engineering Materials, Vol. 5, No. 1 (2017) 1740006 (9 pages)] \\ Chew Ee Wong \\ School of Science, Monash University Malaysia \\ Jalan Lagoon Selatan, 47500 Bandar Sunway, Selangor, Malaysia \\ cewon1@student.monash.edu \\ Anton V. Dolzhenko \\ School of Pharmacy, Monash University Malaysia \\ Jalan Lagoon Selatan, 47500 Bandar Sunway, Selangor, Malaysia \\ Anton.dolzhenko@monash.edu \\ Sui Mae Lee \\ School of Science, Monash University Malaysia \\ Jalan Lagoon Selatan, 47500 Bandar Sunway, Selangor, Malaysia \\ lee.sui.mae@monash.edu \\ David James Young \\ Faculty of Science, Health, Education and Engineering \\ University of the Sunshine Coast, Maroochydore \\ Queensland 4558, Australia \\ dyoung1@usc.edu.au
}

Received 22 June 2017

Accepted 10 July 2017

Published 31 August 2018

The author would like to add the following acknowledgement section in the published article.

\section{Acknowledgements}

We gratefully acknowledge financial support from the Malaysian Ministry of Higher Education under Fundamental Research Grant Scheme (FRGS) (Grant number: FRGS/1/2014/SG06/MUSM/01/1). 